\title{
Atomic-precision measurement of individual atom positions in defects by aberration corrected HRTEM
}

\author{
L. Houben, A. Thust, and K. Urban
}

Ernst Ruska-Centre for Microscopy and Spectroscopy with Electrons (ER-C) and Institute of Solid State Research, Research Centre Jülich. 52425 Jülich, Germany

The combination of imaging in a spherical-aberration corrected transmission electron microscope [1-2] with subsequent numerical focal series reconstruction of the exit-plane wave function (EPW) [3-4] is applied to achieve a reliable quantification of the position of individual atomic columns in defects. An approach for the determination of individual atomic column positions which allows the quantification of the measurement accuracy and is appropriate for non-periodic defects is presented. The approach is based on a regression analysis of peak maxima in the directly interpretable phase image of the EPW retrieved from a focal series of images of a sufficiently thin object. While systematic errors in the positional data are minimized by the compensation of residual aberrations from the EPW, the statistical error due to residual noise in the EPW can be assessed for single, unique atomic columns via the regression analysis.

Figs. 1-3 show the application in the case of a $90^{\circ}$ [100] tilt grain boundary in $\mathrm{YBa}_{2} \mathrm{Cu}_{3} \mathrm{O}_{7-\delta}$ (YBCO), where individual positions in the cation and the oxygen sublattice were determined. Fig. 1 displays the EPW retrieved from a focal series taken in a Philips CM200 FEG ST sphericalaberration corrected microscope [2]. The images were acquired at negative spherical aberration to benefit from an enhanced signal of light elements [7]. Residual aberrations are removed from the EPW, resulting in an excellent pattern match with simulated data and a conservation of the symmetry properties of the basic structure in periodic regions. To account for the overlap of neighbouring columns, peak positions for oxygen columns were measured by a multiple Gaussian least-squares fit of phase profiles (Fig. 2a). The regression analysis typically yields a $2 \sigma$ error radius of $6 \mathrm{pm}$ for oxygen column positions in the periodic parts, increasing to about $20 \mathrm{pm}$ in the grain boundary due to closer spaced columns. For the stronger signal of cation columns $2 \sigma$ error radii of 2 $\mathrm{pm}$ and $4 \mathrm{pm}$ are found respectively. Fig. 3 highlights the individual columns displacements in the reconstructed structure of the grain boundary. Fig. $2 \mathrm{~b}$ displays the bondlengths between the plane copper $\mathrm{Cu} 2$, the chain copper $\mathrm{Cu} 1$ and the apical oxygen $\mathrm{O} 1$, which are the relevant distances correlated with a charge transfer between the copper planes and the superconducting properties [5]. An excellent match with neutron scattering data from ref. [5] is found in the periodic part, whereas a change of these bondlengths is measured with statistical significance in the grain boundary.

Complemented with the sensitivity enhancement for light elements and the possibility of measuring the oxygen occupancy of individual lattice sites offered by the aberration correction [6-7], the atomistic study of the local oxygen coordination at defects in oxide materials can thus provide realistic input data for modelling and understanding electronic-structure relations in these materials.

References

[1] M. Haider et al., Nature 392 (1998) 768.

[2] M. Lentzen et al., Ultramicroscopy 92 (2002) 233.

[3] W. Coene et al., Ultramicroscopy 64 (1996) 109.

[4] A. Thust et al., Ultramicroscopy 64 (1996) 211. 
[5] R.J. Cava et al., Physica C 165 (1990) 419.

[6] C. Kisielowski et al., Ultramicroscopy 89 (2001) 243.

[7] C.L. Jia, M. Lentzen, K. Urban, Science 299 (2003) 870.
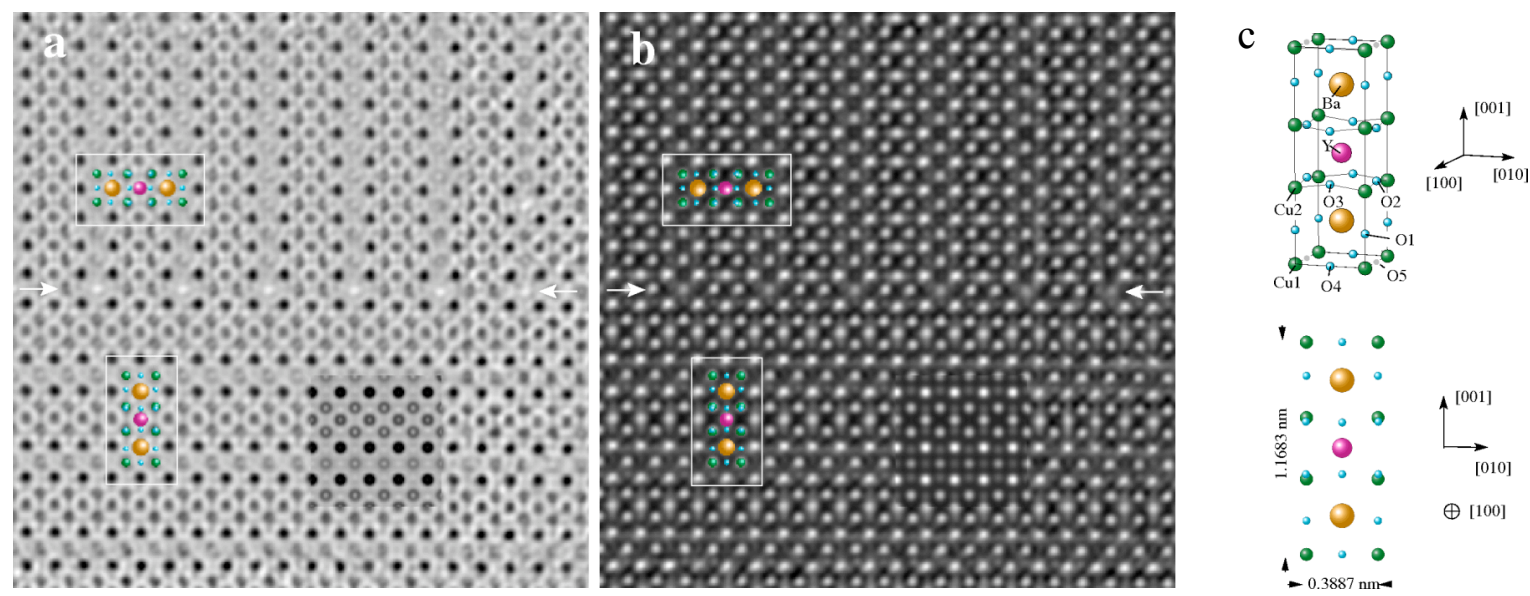

Fig. 1: (a) Amplitude and (b) phase of the EPW of a $90^{\circ}$ boundary in YBCO projected along the [100] direction. The grain boundary plane is marked between arrows. Projections of the elementary unit cell are shown in the boxed regions. Simulated amplitude and phase images are superimposed in the lower right. (c) Unit cell of orthorhombic YBCO and a projection along [100].
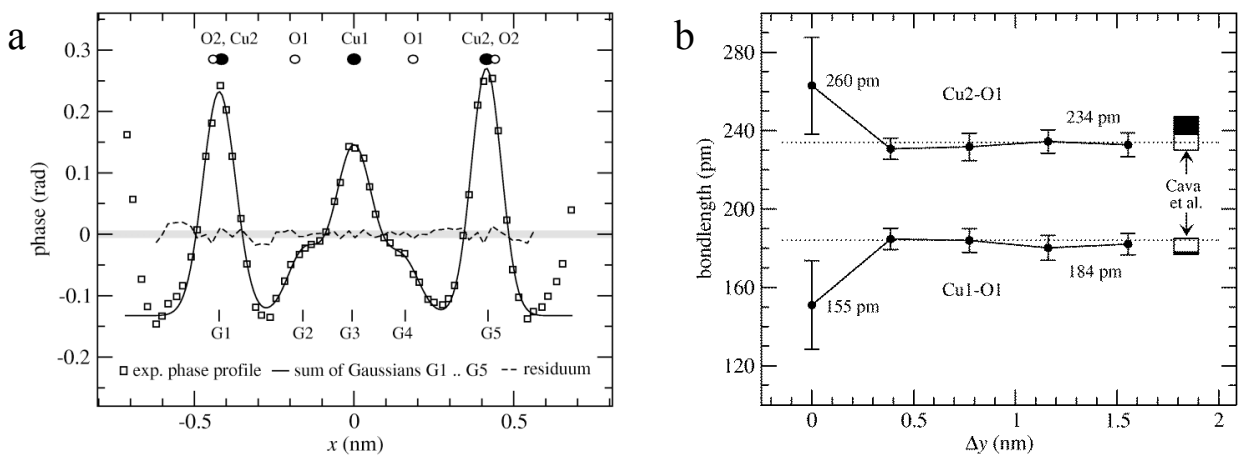

Fig. 2: (a) Least-squares regression of a phase profile along the grain boundary by Gaussian curves, centred at the atomic positions. The gray shaded band represents the $\pm 1 \sigma$-width for the residual noise in the phase of the EPW. (b) Measured bondlengths Cu2-O1 and Cu1-O1 as a function of the distance to the grain boundary in the upper domain. The open (filled) rectangles indicate the range of bondlengths for bulk orthorhombic (tetragonal) YBCO according to ref. [5].

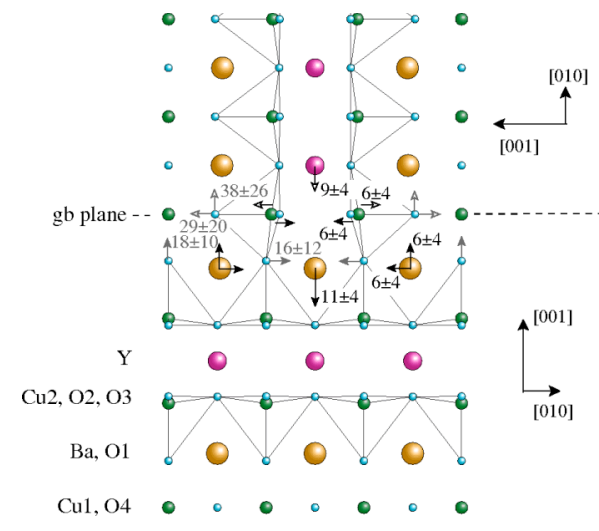

Fig. 3: Individual atom column displacements summarized in a geometrical model of the boundary. Displacements are given with their $2 \sigma$ error radii relative to the lower domain (filled arrows) or the upper domain (open arrows). Polygon lines highlight the pyramidal coordination of the plane copper. 